\title{
Two-Dimensional Gas Chromatography Characterization of Pyrolysis Bio-oils: A Review
}

\section{Supporting Information 1}

\author{
Martin Staš, ${ }^{1 *}$ Miloš Auersvald, ${ }^{1}$ Petr Vozka ${ }^{2}$
}

${ }^{1}$ Department of Petroleum Technology and Alternative Fuels, University of Chemistry and Technology Prague, Technická 5, 16628 Prague, the Czech Republic ${ }^{2}$ Department of Chemistry and Biochemistry, California State University, Los Angeles, CA 90032, USA 
Table S1a: AW: aspen wood, BS: barley straw, BeW: beech wood, BiW: birch wood, CBC: castor been cake, CBS: castor been stalks, CSC: castor seed cake, EW: eucalyptus wood, FR: forestry residue, J: jatropha, MA: microalgae, M: miscanthus, MW: maple wood NS: not specified, OW: oak wood, PW: pine wood, PP: peach pit, SB: sugarcane bagasse, SCS: sugar cane straw, SW: spruce wood, RC: rapeseed cake, RH: rice husks, WS: wheat straw, 


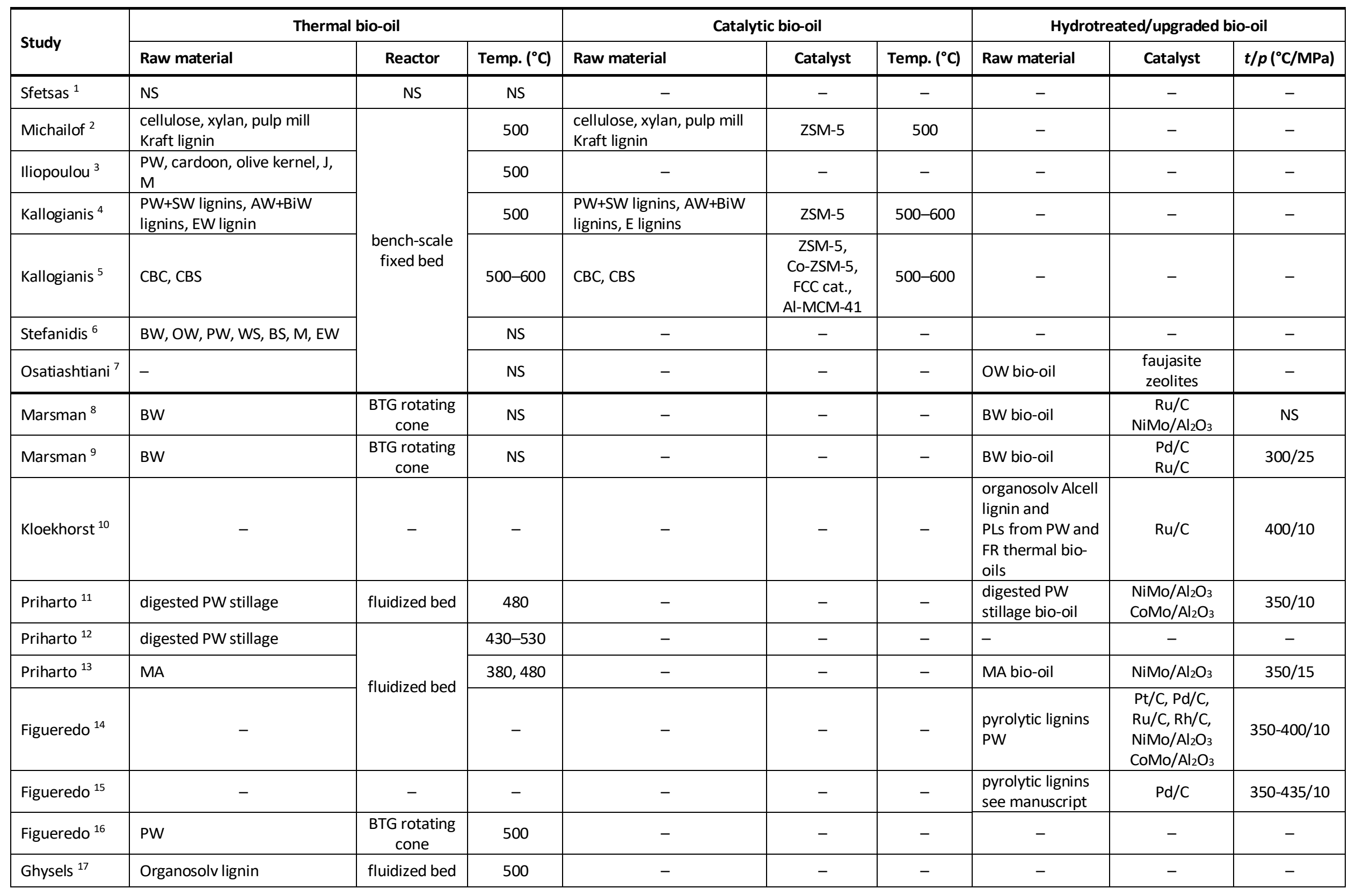


Table S1b: AW: aspen wood, BS: barley straw, BeW: beech wood, BiW: birch wood, CBC: castor been cake, CBS: castor been stalks, CSC: castor seed cake, EW: eucalyptus wood, FR: forestry residue, J: jatropha, MA: microalgae, M: miscanthus, MW: maple wood NS: not specified, OW: oak wood, PW: pine wood, PP: peach pit, SB: sugarcane bagasse, SCS: sugar cane straw, SW: spruce wood, RC: rapeseed cake, RH: rice husks, WS: wheat straw,

\begin{tabular}{|c|c|c|c|c|c|c|c|c|c|}
\hline \multirow{2}{*}{ Study } & \multicolumn{3}{|c|}{ Thermal bio-oil } & \multicolumn{3}{|c|}{ Catalytic bio-oil } & \multicolumn{3}{|c|}{ Hydrotreated bio-oil } \\
\hline & Raw material & Reactor & Temp. $\left({ }^{\circ} \mathrm{C}\right)$ & Raw material & Catalyst & Temp. $\left({ }^{\circ} \mathrm{C}\right)$ & Raw material & Catalyst & $t / p\left({ }^{\circ} \mathrm{C} / \mathrm{MPa}\right)$ \\
\hline Djokic ${ }^{18}$ & PW & - & - & - & - & - & PW bio-oil & $\mathrm{Ru} / \mathrm{C}$ & NS \\
\hline Negahdar ${ }^{19}$ & PW, WS, RC & rotating cone & $480-550$ & - & - & - & - & - & - \\
\hline Toraman 20 & MA & mixed fluid bed & 480 & - & - & - & - & - & - \\
\hline Tessarolo $^{21,22}$ & $\begin{array}{l}\text { empty palm fruit bunch, } \\
\text { PW }\end{array}$ & rotating cone & - & - & - & - & - & - & - \\
\hline Tessarolo $^{23}$ & PW, SB & $\begin{array}{c}\text { circulating fluidized } \\
\text { bed }\end{array}$ & $450-550$ & PW, SB & ZSM-5 & $450-550$ & - & - & - \\
\hline Silva ${ }^{24}$ & $\mathrm{CSC}$ & batch scale, slow pyr. & 380 & - & - & - & - & - & - \\
\hline Silva ${ }^{25}$ & PW & BTG rotating cone & 500 & PW & ZSM-5 & & PW bio-oil & - & - \\
\hline Mendes ${ }^{26}$ & - & - & - & $\mathrm{SB}, \mathrm{PW}$ & ZSM-5 & $450-550$ & - & - & - \\
\hline Moraes ${ }^{27}$ & $\mathrm{RH}, \mathrm{PP}$ & $\begin{array}{l}\text { bench-scale fixed bed } \\
\text { reactor, } \\
\text { intermediate } \\
\text { pyrolysis } \\
\end{array}$ & 700 & - & - & - & - & - & - \\
\hline Moraes ${ }^{28}$ & SCS & \multirow{8}{*}{$\begin{array}{l}\text { bench-scale fixed bed } \\
\text { reactor, } \\
\text { intermediate } \\
\text { pyrolysis } \\
\text { bench-scale bubbling } \\
\text { fluidized bed reactor }\end{array}$} & \multirow[b]{2}{*}{$\begin{array}{c}700 \\
350-550\end{array}$} & - & - & - & - & - & - \\
\hline Faccini ${ }^{29}$ & $\begin{array}{l}\text { EW sawdust, Kraft digester } \\
\text { residue, wastewater } \\
\text { treatment plant sludge }\end{array}$ & & & - & - & - & - & - & - \\
\hline Onorevoli ${ }^{30}$ & $\begin{array}{l}\text { energetic tobacco residual } \\
\text { cake }\end{array}$ & & 700 & - & - & - & - & - & - \\
\hline Primaz ${ }^{31}$ & spent coffee grounds & & $400-600$ & - & - & - & - & - & - \\
\hline Schena ${ }^{32,33}$ & coconut fiber & & 600 & - & - & - & - & - & - \\
\hline Lazzari $^{34}$ & rice husks & & 650 & - & - & - & - & - & - \\
\hline Torri $^{35}$ & EW, SW & & $400-700$ & - & - & - & - & - & - \\
\hline Torri $^{35}$ & EW, SW & & 500 & EW, SW & ZSM-5 & 500 & - & - & - \\
\hline da Cunha ${ }^{36}$ & SCS & NS & NS & - & - & - & - & - & - \\
\hline Maciel $^{37}$ & SCS & & NS & - & - & - & - & - & - \\
\hline Schneider ${ }^{38}$ & from forest timber & fluidized bed & NS & - & - & - & - & - & - \\
\hline
\end{tabular}


Table S1c: AW: aspen wood, BS: barley straw, BeW: beech wood, BiW: birch wood, CBC: castor been cake, CBS: castor been stalks, CSC: castor seed cake, EW: eucalyptus wood, FR: forestry residue, J: jatropha, MA: microalgae, M: miscanthus, MW: maple wood NS: not specified, OW: oak wood, PW: pine wood, PP: peach pit, SB: sugarcane bagasse, SCS: sugar cane straw, SS: sewage sludge, SW: spruce wood, RC: rapeseed cake, RH: rice husks, RS: rice straw, WS: wheat straw

\begin{tabular}{|c|c|c|c|c|c|c|c|c|c|}
\hline \multirow{2}{*}{ Study } & \multicolumn{3}{|c|}{ Thermal bio-oil } & \multicolumn{3}{|c|}{ Catalytic bio-oil } & \multicolumn{3}{|c|}{ Hydrotreated bio-oil } \\
\hline & Raw material & Reactor & Temp. $\left({ }^{\circ} \mathrm{C}\right)$ & Raw material & Catalyst & Temp. $\left({ }^{\circ} \mathrm{C}\right)$ & Raw material & Catalyst & $t / p\left({ }^{\circ} \mathrm{C} / \mathrm{MPa}\right)$ \\
\hline Omais ${ }^{39}$ & - & - & - & - & - & - & Not specified & Not specified & Not specified \\
\hline Eschenbacher ${ }^{40,41}$ & WS & $\begin{array}{l}\text { Bench scale, } \\
\text { ablative }\end{array}$ & $350-530$ & WS & $\begin{array}{c}\mathrm{Na} / \mathrm{Al}_{2} \mathrm{O}_{3} \\
\mathrm{Mo} / \mathrm{Al}_{2} \mathrm{O}_{3} \\
\mathrm{MoO}_{3} / \mathrm{TiO}_{2} \\
\mathrm{Pt} / \mathrm{TiO}_{2} \\
\end{array}$ & $350-530$ & - & - & - \\
\hline Karp ${ }^{42}$ & ow & $\begin{array}{l}\text { Entrained } \\
\text { flow reactor }\end{array}$ & 500 & - & - & - & - & - & - \\
\hline Johansson ${ }^{43}$ & FR, PW, salix & $\begin{array}{l}\text { Cyclone } \\
\text { pyrolysis }\end{array}$ & 750 & - & - & - & - & - & - \\
\hline Fullana $^{44}$ & C, L, SS & quartz tube & 700 & - & - & - & - & - & - \\
\hline Rathsack ${ }^{45}$ & See the manuscript & - & - & - & - & - & - & - & - \\
\hline Ware ${ }^{46}$ & PW & $\begin{array}{l}\text { cubical } \\
\text { batch }\end{array}$ & 500 & - & - & - & - & - & - \\
\hline Mao $^{47}$ & - & $\begin{array}{l}\text { bubbling } \\
\text { bed }\end{array}$ & - & Corn cob & ZSM-5 & $500-600$ & - & - & - \\
\hline Wang ${ }^{48}$ & RS & $\begin{array}{l}\text { fluidized } \\
\text { bed }\end{array}$ & 500 & - & - & - & - & - & - \\
\hline Mullen et al. ${ }^{49}$ & $\begin{array}{l}\text { pennycress } \\
\text { presscake }\end{array}$ & $\begin{array}{l}\text { fluidized } \\
\text { bed }\end{array}$ & - & - & - & - & $\begin{array}{l}\text { pennycress } \\
\text { presscake }\end{array}$ & $\begin{array}{l}\mathrm{Ru} / \mathrm{C}, \mathrm{Pd} / \mathrm{C}, \\
\mathrm{Pt} / \mathrm{C}\end{array}$ & $320 / 13.8$ \\
\hline Margeriat et al. ${ }^{50}$ & BW & - & - & BW & $\begin{array}{c}\text { Several } \\
\text { modified } \\
\text { zeolite based }\end{array}$ & 500 & - & - & - \\
\hline Hung et al. ${ }^{51}$ & cellulose & - & $400-600$ & - & - & - & - & - & - \\
\hline $\begin{array}{l}\text { Shashkov and } \\
\text { Sidelnikov } 52\end{array}$ & NS & NS & NS & - & - & - & - & - & - \\
\hline Olcese $^{53}$ & lignin & tubular & 500 & - & $\begin{array}{c}\mathrm{Fe} / \mathrm{SiO}_{2} \\
\mathrm{Fe} / \mathrm{C}\end{array}$ & 400 & - & - & - \\
\hline
\end{tabular}


Table S2a: Group of A. Lappas

\begin{tabular}{|c|c|c|}
\hline & Sfetsas et al. ${ }^{1}$ & $\begin{array}{l}\text { Michailof et al. }^{2} \\
\text { Iliopoulou et al. }^{3} \\
\text { Kalogiannis et al. }{ }^{4,5} \\
\text { Stefanidis et al. }^{6} \\
\text { Osatiashtiani et al. }{ }^{7}\end{array}$ \\
\hline Published & 2011 & 2014-2017 \\
\hline Method & $\mathrm{GC} \times \mathrm{GC}-\mathrm{TOF}-\mathrm{MS}$ & $\mathrm{GC} \times \mathrm{GC}-\mathrm{TOF}-\mathrm{MS}$ \\
\hline Bio-oils & Three different non-specified thermal bio-oils. & Various thermal and catalytic, see Table S1 \\
\hline Sample treatment & $\begin{array}{l}\text { Diluted in acetone and then filtered through } \\
\text { a membrane filter with a pore diameter of } 0.45 \mu \mathrm{m} \text {. }\end{array}$ & Diluted in methanol. \\
\hline Internal standard & no & Phenol- $d_{6}(50 \mu \mathrm{g} / \mathrm{g})$ \\
\hline Gas chromatograph & 7890 A (Agilent) & 7890 A (Agilent) \\
\hline Carrier gas & helium (5.0) & helium (5.0) \\
\hline Carrier gas flow rate & $1 \mathrm{~mL} / \mathrm{min}$ (constant flow) & $1 \mathrm{~mL} / \mathrm{min}$ (constant flow) \\
\hline Injector temperature & $250^{\circ} \mathrm{C}$ & $250^{\circ} \mathrm{C}$ \\
\hline Injection volume & $0.2 \mu \mathrm{L}$ & $0.5 \mu \mathrm{L}$ \\
\hline Split injection ratio & $1: 40$ & $1: 20$ \\
\hline Temperature program & $\begin{array}{l}35^{\circ} \mathrm{C}(10 \mathrm{~min}) \text {, then } 3^{\circ} \mathrm{C} / \mathrm{min} \text { to } 250^{\circ} \mathrm{C} \text {, then } \\
15^{\circ} \mathrm{C} / \mathrm{min} \text { to } 300^{\circ} \mathrm{C}(15 \mathrm{~min}) \text {. } \\
\text { The secondary oven was programmed } 15^{\circ} \mathrm{C} \text { ahead } \\
\text { of the primary } \mathrm{GC} \text { oven gradient. }\end{array}$ & $\begin{array}{l}35^{\circ} \mathrm{C}(10 \mathrm{~min}) \text {, then } 3{ }^{\circ} \mathrm{C} / \mathrm{min} \text { to } 250^{\circ} \mathrm{C}(10 \mathrm{~min}) \text {, then } 15 \\
{ }^{\circ} \mathrm{C} / \mathrm{min} \text { to } 330^{\circ} \mathrm{C}(15 \mathrm{~min}) \text {. } \\
\text { The secondary oven was programmed } 15{ }^{\circ} \mathrm{C} \text { ahead of the } \\
\text { primary GC oven gradient. }\end{array}$ \\
\hline Columns & $\begin{array}{l}1^{\text {st }} \text { D: VF-5MS } \\
\text { (5\% phenyl, } 95 \% \text { PDMS }) \\
(30 \mathrm{~m} \times 0.25 \mathrm{~mm} \times 0.25 \mu \mathrm{m}) \\
2^{\text {nd }} \text { D: VF-17MS } \\
(50 \% \text { phenyl, } 50 \% \text { PDMS }) \\
(1.65 \mathrm{~m} \times 0.10 \mathrm{~mm} \times 0.20 \mu \mathrm{m})\end{array}$ & $\begin{array}{l}1^{\text {st }} \text { D: BPX-5 } \\
(5 \% \text { phenyl, } 95 \% \text { PSPS }) \\
(30 \mathrm{~m} \times 0.25 \mathrm{~mm} \times 0.25 \mu \mathrm{m}) \\
2^{\text {nd }} \text { D: BPX-50 } \\
(50 \% \text { phenyl, } 50 \% \text { polysilphenylene-siloxane }) \\
(1.5 \mathrm{~m} \times 0.10 \mathrm{~mm} \times 0.10 \mu \mathrm{m})\end{array}$ \\
\hline Ovens & 2 & 2 \\
\hline Modulation time (s) & 10 & 5 \\
\hline Modulator & liquid $\mathrm{N}_{2}$ quad jet dual stage modulator (Zoex) & liquid $\mathrm{N}_{2}$ quad jet dual stage modulator (Zoex) \\
\hline $\begin{array}{l}\text { Modulator } \\
\text { temperature offset }\end{array}$ & $30^{\circ} \mathrm{C}$ & $30^{\circ} \mathrm{C}$ \\
\hline Mass spectrometer & Pegasus 4D (Leco Instruments) & Pegasus 4D (Leco Instruments) \\
\hline Mass analyzer & TOF & TOF \\
\hline Scanning range $(m / z)$ & $45-450$ & $45-450$ \\
\hline Acquisition rate $(\mathrm{Hz})$ & 100 & 100 \\
\hline Quantification & 11 compounds - TOF-MS - EIC & 39 compounds, TOF-MS - RRFs \\
\hline
\end{tabular}


Table S2b Group of H. J. Heeres

\begin{tabular}{|c|c|c|}
\hline & Marsman et al. ${ }^{8}$ & Marsman et al. ${ }^{9}$ \\
\hline Published & 2007 & 2008 \\
\hline Method & $\mathrm{GC} \times \mathrm{GC}-\mathrm{FID}$ & $\mathrm{GC} \times \mathrm{GC}-\mathrm{TOF}-\mathrm{MS}$ \\
\hline Column configuration & NP & NP \\
\hline Bio-oils & $\begin{array}{l}\text { Thermal BW bio-oil from BTG rotating cone } \\
\text { technology. } \\
\text { One HBO produced thereof. }\end{array}$ & $\begin{array}{l}\text { Thermal BW bio-oil from BTG rotating cone } \\
\text { technology. } \\
\text { Two HBOs produced thereof. }\end{array}$ \\
\hline Sample treatment & dilution with THF $(1: 1$, wt.) & dilution with THF $(1: 1$, wt. $)$ \\
\hline Internal standard & n-decane & n-decane \\
\hline Gas chromatograph & Trace GC (Thermo-Fisher Scientific) & HP 6890 N (Agilent) \\
\hline Carrier gas & helium (5.0) & helium (5.0) \\
\hline Carrier gas flow rate & $0.6 \mathrm{~mL} / \mathrm{min}$ (constant flow) & $1 \mathrm{~mL} / \mathrm{min}$ (constant flow) \\
\hline Injector temperature & $300^{\circ} \mathrm{C}$ & $275^{\circ} \mathrm{C}$ \\
\hline Injection volume & $1 \mu \mathrm{L}$ & $0.1 \mu \mathrm{L}$ \\
\hline Split injection ratio & $1: 500$ & $1: 10$ \\
\hline Temperature program & $40{ }^{\circ} \mathrm{C}(5 \mathrm{~min})$, then $3{ }^{\circ} \mathrm{C} / \mathrm{min}$ to $300^{\circ} \mathrm{C}$ & $\begin{array}{l}40{ }^{\circ} \mathrm{C}(5 \mathrm{~min}) \text {, then } 3{ }^{\circ} \mathrm{C} / \mathrm{min} \text { to } 330^{\circ} \mathrm{C} \\
\text { The secondary oven was programmed } 10^{\circ} \mathrm{C} \\
\text { ahead of the primary } \mathrm{GC} \text { oven gradient. }\end{array}$ \\
\hline Columns & $\begin{array}{l}1^{\text {st }} \mathrm{D}: \text { SolGel }(100 \% \text { PDMS }) \\
(30 \mathrm{~m} \times 0.25 \mathrm{~mm} \times 0.25 \mu \mathrm{m}) \\
2^{\text {nd }} \mathrm{D}: \text { OV-1710 } \\
(50 \% \text { phenyl, } 50 \% \text { PDMS }) \\
(1.5 \mathrm{~m} \times 0.10 \mathrm{~mm} \times 0.10 \mu \mathrm{m})\end{array}$ & $\begin{array}{l}1^{\text {st }} \text { D: VF-5MS } \\
\text { (5\% phenyl, } 95 \% \text { PSPS }) \\
(30 \mathrm{~m} \times 0.25 \mathrm{~mm} \times 0.25 \mu \mathrm{m}) \\
2^{\text {nd }} \text { D: VF-17MS } \\
(50 \% \text { phenyl, } 50 \% \text { PDMS }) \\
(2 \mathrm{~m} \times 0.10 \mathrm{~mm} \times 0.20 \mu \mathrm{m})\end{array}$ \\
\hline Ovens & 2 & 2 \\
\hline Modulation time (s) & 6 & 10 \\
\hline Modulation & liquid $\mathrm{CO}_{2}$ dual jet modulator & liquid $\mathrm{N}_{2}$ quad jet modulator (Zoex) \\
\hline Detector & FID & Pegasus III (Leco Instruments) \\
\hline Detector temp. & $300^{\circ} \mathrm{C}$ & - \\
\hline Mass analyzer & - & TOF \\
\hline Ionization (temp.) & - & not specified \\
\hline Scanning range $(m / z)$ & - & $50-500$ \\
\hline Acquisition rate $(\mathrm{Hz})$ & - & 100 \\
\hline Quantification & RPV & RPV \\
\hline
\end{tabular}


Table S2b Group of H. J. Heeres (continued)

\begin{tabular}{|c|c|c|}
\hline & $\begin{array}{l}\text { Kloekhorst et al. }{ }^{10} \\
\text { Priharto et al. }{ }^{11-13}\end{array}$ & $\begin{array}{l}\text { Figueiredo et al. }{ }^{14-16} \\
\text { Ghysels et al. }{ }^{17}\end{array}$ \\
\hline Published & $2014-2020$ & $2019-2020$ \\
\hline Method & $\mathrm{GC} \times \mathrm{GC}-\mathrm{FID}$ & $\begin{array}{l}\text { GC } \times \text { GC-FID (see the previous column) } \\
\mathrm{GC} \times \mathrm{GC}-\mathrm{TOF}-\mathrm{MS}\end{array}$ \\
\hline Bio-oils & $\mathrm{RP}$ & $\mathrm{RP}$ \\
\hline Sample treatment & $\begin{array}{l}\text { The sample was diluted to a } \\
5 \% \text { vol. solution using GC-grade } \\
\text { tetrahydrofuran (Sigma-Aldrich), and an } \\
\text { internal standard was added. The diluted } \\
\text { sample was filtered using a PTFE syringe filter } \\
(0.2 \mu \mathrm{m} \text { pore size) before injection. }\end{array}$ & - \\
\hline Internal standard & di-n-butyl ether (1 g/L) & di-n-butyl ether (500 mg/L) \\
\hline Gas chromatograph & 7890 B (Agilent) & 7890 B (Agilent) \\
\hline Carrier gas & helium (5.0) & helium (4.5) \\
\hline Carrier gas flow rate & $\begin{array}{l}0.6 \mathrm{~mL} / \mathrm{min} \text { (constant flow) } \\
0.8 \mathrm{~mL} / \mathrm{min} \text { (constant flow) }\end{array}$ & $0.8 \mathrm{~mL} / \mathrm{min}$ (constant flow) \\
\hline Injector temperature & $250^{\circ} \mathrm{C}$ & $280^{\circ} \mathrm{C}$ \\
\hline Injection volume & - & $1 \mu \mathrm{L}$ \\
\hline Split injection ratio & - & $1: 50$ \\
\hline Temperature program & $\begin{array}{l}40{ }^{\circ} \mathrm{C}(5 \mathrm{~min}) \text {, then } 3{ }^{\circ} \mathrm{C} / \mathrm{min} \text { to } 250^{\circ} \mathrm{C} \\
\text { The secondary oven was programmed } 10^{\circ} \mathrm{C} \\
\text { ahead of the primary } \mathrm{GC} \text { oven gradient. }\end{array}$ & $\begin{array}{l}\text { Oven } 1: 40^{\circ} \mathrm{C}(5 \mathrm{~min}) \text {, then } 3{ }^{\circ} \mathrm{C} / \mathrm{min} \text { to } 250^{\circ} \mathrm{C} \\
\text { Oven } 2:-50{ }^{\circ} \mathrm{C}(45 \mathrm{~min}) \text {, then } 20^{\circ} \mathrm{C} / \mathrm{min} \text { to } \\
9^{\circ} \mathrm{C}\end{array}$ \\
\hline Columns & $\begin{array}{l}1^{\text {st }} \text { D: } \mathbf{R T X - 1 7 0 1} \\
(14 \% \mathrm{CPP}, 86 \% \text { PDMS }) \\
(30 \mathrm{~m} \times 0.25 \mathrm{~mm} \times 0.25 \mu \mathrm{m}) \\
2^{\text {nd }} \mathrm{D}: \text { Rxi-5Sil-MS } \\
(1,4 \text {-bis(dimethylsiloxy)phenylene dimethyl } \\
\text { polysiloxane) } \\
(1.2 \mathrm{~m} \times 0.15 \mathrm{~mm} \times 0.15 \mu \mathrm{m})\end{array}$ & $\begin{array}{l}1^{\text {st }} \text { D: RTX-1701 } \\
(14 \% \text { CPP, } 86 \% \text { PDMS }) \\
(30 \mathrm{~m} \times 0.25 \mathrm{~mm} \times 0.25 \mu \mathrm{m}) \\
2^{\text {nd }} \text { D: Rxi-5Sil-MS } \\
(50 \% \text { phenyl, } 50 \% \text { PDMS }) \\
(1.2 \mathrm{~m} \times 0.15 \mathrm{~mm} \times 0.15 \mu \mathrm{m})\end{array}$ \\
\hline Ovens & 2 & 2 \\
\hline Modulation time (s) & 6 & 6 \\
\hline Modulator & liquid $\mathrm{CO}_{2}$ dual jet modulator & liquid $\mathrm{CO}_{2}$ dual jet modulator \\
\hline Detector & FID & FID, MS \\
\hline Detector temp. & $250^{\circ} \mathrm{C}$ & $280^{\circ} \mathrm{C}$ (FID) \\
\hline Mass analyzer & - & TOF \\
\hline Ionization (temp.) & - & $\mathrm{El},+70 \mathrm{eV}\left(280^{\circ} \mathrm{C}\right)$ \\
\hline Scanning range $(m / z)$ & - & - \\
\hline Acquisition rate & - & - \\
\hline Quantification & Average RRFs for component groups (FID) & Average RRFs for component groups (FID) \\
\hline
\end{tabular}


Table S2c: Group of K. M. Van Geem

\begin{tabular}{|c|c|c|c|}
\hline & Djokic et al. ${ }^{18}$ & Negahdar et al. ${ }^{19}$ & Toraman et al. ${ }^{20}$ \\
\hline Published & 2012 & 2016 & 2016 \\
\hline Method & $\mathrm{GC} \times \mathrm{GC}-\mathrm{TOF}-\mathrm{MS} / \mathrm{FID}$ & $\begin{array}{l}\mathrm{GC} \times \mathrm{GC}-\mathrm{TOF}-\mathrm{MS} \\
\mathrm{GC} \times \mathrm{GC}-\mathrm{FID}\end{array}$ & $\begin{array}{l}\mathrm{GC} \times \mathrm{GC}-\mathrm{TOF}-\mathrm{MS} \\
\mathrm{GC} \times \mathrm{GC}-\mathrm{NCD}\end{array}$ \\
\hline $\begin{array}{l}\text { Column } \\
\text { configuration }\end{array}$ & $\begin{array}{l}\mathrm{NP} \\
\mathrm{RP}\end{array}$ & NP & $\begin{array}{l}\mathrm{NP} \\
\mathrm{RP}\end{array}$ \\
\hline Bio-oils & $\begin{array}{l}\text { thermal PW bio-oil } \\
\text { HDO produced thereof }\end{array}$ & $\begin{array}{l}\text { three different thermal bio-oils (PW, } \\
\mathrm{WS}, \mathrm{RC} \text { ), rotating cone technology }\end{array}$ & $\begin{array}{l}\text { organic and aqueous phase (OP and } \\
\text { AP) of a microalgae bio-oil }\end{array}$ \\
\hline Sample treatment & dilution with THF (1:1, wt.) & dilution with THF (1:1, wt.) & $\begin{array}{l}\text { OP: dilution with THF }(2: 1, \text { wt. }) \\
\text { AP: no dilution }\end{array}$ \\
\hline Internal standard & dibutyl ether and fluoranthene & dibutyl ether and fluoranthene & NCD: caprolactam (0.5 wt. \%) \\
\hline Gas chromatograph & Trace GC (Thermo-Fisher Scientific) & Trace GC (Thermo-Fisher Scientific) & Trace GC (Thermo-Fisher Scientific) \\
\hline Carrier gas & helium & helium & helium \\
\hline Carrier gas flow rate & $\begin{array}{l}\text { MS: } 3.5 \mathrm{~mL} / \mathrm{min} \text { (constant flow) } \\
\text { FID: } 2.1 \mathrm{~mL} / \mathrm{min} \text { (constant flow) }\end{array}$ & $\begin{array}{l}\text { MS: see the manuscript } \\
\text { FID: } 2.1 \mathrm{~mL} / \mathrm{min} \text { (constant flow) }\end{array}$ & see the manuscript \\
\hline $\begin{array}{l}\text { Injector type } \\
\text { and temperature }\end{array}$ & $280^{\circ} \mathrm{C}$ & $\begin{array}{l}\text { MS: } 300{ }^{\circ} \mathrm{C} \\
\text { FID: see the manuscript }\end{array}$ & $300^{\circ} \mathrm{C}, \mathrm{MS}: \mathrm{SSL}, \mathrm{NCD}: \mathrm{PTV}$ \\
\hline Injection volume & $0.2 \mu \mathrm{L}$ & not specified & $0.2 \mu \mathrm{L}$ \\
\hline Split injection ratio & $1: 75$ & see the manuscript & see the manuscript \\
\hline $\begin{array}{l}\text { Temperature } \\
\text { program }\end{array}$ & $40{ }^{\circ} \mathrm{C}$ then $3{ }^{\circ} \mathrm{C} / \mathrm{min}$ to $300^{\circ} \mathrm{C}$ & see the manuscript & see the manuscript \\
\hline Columns & $\begin{array}{l}\text { Normal column combination: } \\
1^{\text {st }} \mathrm{D}: \text { Rtx-1 PONA }(100 \% \text { PDMS }) \\
(50 \mathrm{~m} \times 0.25 \mathrm{~mm} \times 0.50 \mu \mathrm{m}) \\
2^{\text {nd }} \mathrm{D}: \text { BPX-50 } \\
(50 \% \text { phenyl, } 50 \% \text { polysilphenylene- } \\
\text { siloxane) } \\
(2 \mathrm{~m} \times 0.15 \mathrm{~mm} \times 0.15 \mu \mathrm{m}) \\
\text { Reversed column combination: } \\
1^{\text {st }} \mathrm{D}: \text { BPX-50 } \\
(50 \% \text { phenyl, } 50 \% \text { polysilphenylene- } \\
\text { siloxane }) \\
(60 \mathrm{~m} \times 0.15 \mathrm{~mm} \times 0.15 \mu \mathrm{m}) \\
2^{\text {nd }} \mathrm{D}: \text { Rtx-1 PONA }(100 \% \text { PDMS }) \\
(1 \mathrm{~m} \times 0.25 \mathrm{~mm} \times 0.50 \mu \mathrm{m})\end{array}$ & $\begin{array}{l}\text { Column combination 1: } \\
1^{\text {st }} \mathrm{D}: \mathrm{MXT}-1(100 \% \text { PDMS) } \\
(60 \mathrm{~m} \times 0.25 \mathrm{~mm} \times 0.50 \mu \mathrm{m}) \\
2^{\text {nd }} \mathrm{D}: \text { BPX-50 } \\
(50 \% \text { phenyl, } 50 \% \text { polysilphenylene- } \\
\text { siloxane }) \\
(2 \mathrm{~m} \times 0.15 \mathrm{~mm} \times 0.15 \mu \mathrm{m}) \\
\text { Column combination } 2: \\
1^{\text {st }} \mathrm{D}: \text { Rtx-1 PONA }(100 \% \mathrm{PDMS}) \\
(50 \mathrm{~m} \times 0.25 \mathrm{~mm} \times 0.50 \mu \mathrm{m}) \\
2^{\text {nd }} \mathrm{D}: \text { BPX-50 } \\
(50 \% \text { phenyl, } 50 \% \text { polysilphenylene- } \\
\text { siloxane }) \\
(2 \mathrm{~m} \times 0.15 \mathrm{~mm} \times 0.15 \mu \mathrm{m}) \\
\text { For further details see the } \\
\text { manuscript. }\end{array}$ & $\begin{array}{l}\text { Normal column combination: } \\
1^{\text {st }} \mathrm{D}: \mathrm{MXT}-1(100 \% \text { PDMS }) \\
(60 \mathrm{~m} \times 0.25 \mathrm{~mm} \times 0.50 \mu \mathrm{m}) \\
2^{\text {nd }} \mathrm{D}: \text { BPX-50 } \\
(50 \% \text { phenyl, } 50 \% \text { polysilphenylene- } \\
\text { siloxane }) \\
(2 \mathrm{~m} \times 0.15 \mathrm{~mm} \times 0.15 \mu \mathrm{m}) \\
\text { Reversed column combination: } \\
1^{\text {st }} \mathrm{D}: \text { IL-59 } \\
(30 \mathrm{~m} \times 0.25 \mathrm{~mm} \times 0.50 \mu \mathrm{m}) \\
2^{\text {nd }} \mathrm{D}: \text { Rxi-5ms } \\
(5 \% \text { phenyl, } 95 \% \text { PDMS }) \\
(2 \mathrm{~m} \times 0.10 \mathrm{~mm} \times 0.10 \mu \mathrm{m})\end{array}$ \\
\hline Ovens & 1 & 1 & 1 \\
\hline Modulation time (s) & 7 & 7 & $6-10$, see the manuscript \\
\hline Modulation & $\begin{array}{l}\text { liquid } \mathrm{CO}_{2} \text { two-stage cryogenic } \\
\text { modulator }\end{array}$ & $\begin{array}{l}\text { liquid } \mathrm{CO}_{2} \text { two-stage cryogenic } \\
\text { modulator }\end{array}$ & $\begin{array}{l}\text { liquid } \mathrm{CO}_{2} \text { two-stage cryogenic } \\
\text { modulator }\end{array}$ \\
\hline Detector & FID, MS & FID, MS & $\mathrm{NCD}, \mathrm{MS}$ \\
\hline Detector temp. & $280^{\circ} \mathrm{C}$ (FID) & not specified & NCD: $920^{\circ} \mathrm{C}$ \\
\hline Mass analyzer & TOF & TOF & TOF \\
\hline Ionization (temp.) & not specified & not specified & $200^{\circ} \mathrm{C}$ \\
\hline $\begin{array}{l}\text { Scanning range } \\
(\mathrm{m} / \mathrm{z})\end{array}$ & $35-400$ & $25-500$ & $20-500$ \\
\hline Acquisition rate & \multicolumn{2}{|c|}{ TOF-MS $(30 \mathrm{~Hz})$, FID $(100 \mathrm{~Hz})$} & TOF-MS $(30 \mathrm{~Hz}), \mathrm{NCD}(100 \mathrm{~Hz})$ \\
\hline Quantification & $\begin{array}{l}16 \text { compounds - FID response factor } \\
\text { Other - FID - ECN }\end{array}$ & $\begin{array}{l}16 \text { compounds - FID response factor } \\
\text { Other - FID - ECN }\end{array}$ & NCD \\
\hline
\end{tabular}


Table S2d: Group of D. A. Azevedo

\begin{tabular}{|c|c|c|}
\hline & $\begin{array}{l}\text { Tessarolo et al. }{ }^{21-23} \\
\text { Silva et al. }{ }^{24,25} \\
\text { Mendes et al. }{ }^{26}\end{array}$ & Moraes et al. 27,28 \\
\hline Published & 2013-2017 & 2012 \\
\hline Method & $\mathrm{GC} \times \mathrm{GC}-\mathrm{TOF}-\mathrm{MS}$ & $\mathrm{GC} \times \mathrm{GC}-\mathrm{TOF}-\mathrm{MS}$ \\
\hline Column configuration & NP & NP \\
\hline Sample treatment & Dissolved in DCM (no further details provided) & Dissolved in DCM (no further details provided) \\
\hline Internal standard & No & No \\
\hline Gas chromatograph & $6890 \mathrm{~N}$ (Agilent) & $6890 \mathrm{~N}$ (Agilent) \\
\hline Carrier gas & helium (5.0) & helium (5.0) \\
\hline Carrier gas flow rate & $1 \mathrm{~mL} / \mathrm{min}$ (constant flow) & $1 \mathrm{~mL} / \mathrm{min}$ (constant flow) \\
\hline $\begin{array}{l}\text { Injector type } \\
\text { and temperature }\end{array}$ & $\mathrm{SSL}, 280^{\circ} \mathrm{C}$ & $\begin{array}{l}\text { SSL, } \\
275^{\circ} \mathrm{C} \text { (Moraes FPT) } \\
280^{\circ} \mathrm{C} \text { (Moraes JAAP) }\end{array}$ \\
\hline Split injection ratio & splitless & $1: 10$ \\
\hline Injection volume & $1 \mu \mathrm{L}$ & - \\
\hline Temperature program & $\begin{array}{l}35^{\circ} \mathrm{C}(15 \mathrm{~min}) \text {, then } 4{ }^{\circ} \mathrm{C} / \mathrm{min} \text { to } 330^{\circ} \mathrm{C} \text {. The } \\
\text { secondary oven temperature program was } 20^{\circ} \mathrm{C} \\
\text { higher than the primary one. }\end{array}$ & $\begin{array}{l}40{ }^{\circ} \mathrm{C}(5 \mathrm{~min}) \text {, then } 3{ }^{\circ} \mathrm{C} / \mathrm{min} \text { to } 315^{\circ} \mathrm{C}(10 \mathrm{~min}) . \text { The } \\
\text { secondary column temperature was } 10^{\circ} \mathrm{C} \text { above the } \\
\text { temperature of the main oven }\end{array}$ \\
\hline Columns & $\begin{array}{l}1^{\text {st }} \text { D: DB-5 (5 \% phenyl, } 95 \% \text { PDMS) } \\
(30 \mathrm{~m} \times 0.25 \mathrm{~mm} \times 0.25 \mu \mathrm{m}) \\
2^{\text {nd }} \mathrm{D}: \text { BPX-50 } \\
(50 \% \text { phenyl, } 50 \% \text { PSPS }) \\
(1.5 \mathrm{~m} \times 0.10 \mathrm{~mm} \times 0.10 \mu \mathrm{m}) \\
\text { This column was connected to the TOF via } 0.5 \mathrm{~m} \times \\
0.25 \mathrm{~mm} \text { empty deactivated fused silica capillary } \\
\text { using SGE mini-unions and SilTiteTM } \\
\text { metal ferrules }(0.1-0.25 \mathrm{~mm}) \text {, see the manuscript }\end{array}$ & $\begin{array}{l}1^{\text {st }} \text { D: DB-5 (5 \% phenyl, } 95 \% \text { PDMS) } \\
(30 \mathrm{~m} \times 0.25 \mathrm{~mm} \times 0.25 \mu \mathrm{m}) \\
2^{\text {nd }} \text { D: DB-17 } \mathrm{ms} \\
(50 \% \text { phenyl, } 50 \% \text { PDMS }) \\
(1.2 \mathrm{~m} \times 0.18 \mathrm{~mm} \times 0.18 \mu \mathrm{m}) \text { (Moraes FPT) } \\
\text { DB-17 ms } \\
(50 \% \text { phenyl, } 50 \% \text { PDMS }) \\
(1.84 \mathrm{~m} \times 0.18 \mathrm{~mm} \times 0.18 \mu \mathrm{m}) \text { (Moraes JAAP) }\end{array}$ \\
\hline Ovens & 2 & 2 \\
\hline Modulation time (s) & 6 & $\begin{array}{l}10 \mathrm{~s} \text { with hot and cold } \\
\text { pulse of } 0.4 \mathrm{~s} \text { and } 2.1 \mathrm{~s} \text {, respectively }\end{array}$ \\
\hline Modulation & quad-jet dual-stage modulator & liquid $\mathrm{N}_{2}$ quad-jet modulator \\
\hline Transfer line temp. & $280^{\circ} \mathrm{C}$ & $\begin{array}{l}275^{\circ} \mathrm{C} \text { (Moraes FPT) } \\
280^{\circ} \mathrm{C} \text { (Moraes JAAP) }\end{array}$ \\
\hline Detector & MS & MS \\
\hline Mass analyzer & TOF (Pegasus III, Leco) & TOF (Pegasus IV, Leco) \\
\hline Ionization (temp.) & $\mathrm{EI},+70 \mathrm{eV}, 230^{\circ} \mathrm{C}$ & $\mathrm{EI},+70 \mathrm{eV}, 250^{\circ} \mathrm{C}$ \\
\hline Scanning range $(m / z)$ & $35-600$ & $\begin{array}{l}\text { 45-500 (Moraes FPT) } \\
50-500 \text { (Moraes JAAP) }\end{array}$ \\
\hline Acquisition rate $(\mathrm{Hz})$ & $100 \mathrm{~Hz}$ & $100 \mathrm{~Hz}$ \\
\hline Quantification & RPV & RPV \\
\hline
\end{tabular}


Table S2d: Group of D. A. Azevedo (continued)

\begin{tabular}{|c|c|c|}
\hline & $\begin{array}{l}\text { da Cunha et al. }{ }^{36} \\
\text { Schneider et al. }{ }^{38}\end{array}$ & $\begin{array}{l}\text { Faccini et al. }{ }^{29} \\
\text { Maciel et al- }{ }^{37}\end{array}$ \\
\hline Published & 2013-2014 & 2013 \\
\hline Method & $\mathrm{GC} \times \mathrm{GC}-\mathrm{q}-\mathrm{MS}$ & $\mathrm{GC} \times \mathrm{GC}-\mathrm{TOF}-\mathrm{MS}$ \\
\hline $\begin{array}{l}\text { Column } \\
\text { configuration }\end{array}$ & NP & NP \\
\hline $\begin{array}{l}\text { Sample } \\
\text { treatment }\end{array}$ & - & - \\
\hline Internal standard & - & - \\
\hline $\begin{array}{l}\text { Gas } \\
\text { chromatograph }\end{array}$ & QP2010 Ultra (Shimadzu) & $6890 \mathrm{~N}$ (Agilent) \\
\hline Carrier gas & helium (5.0) & helium (5.0) \\
\hline $\begin{array}{l}\text { Carrier gas flow } \\
\text { rate }\end{array}$ & $\begin{array}{l}0.89 \mathrm{~mL} / \mathrm{min} \text { (constant flow), da Cunha } \\
0.91 \mathrm{~mL} / \mathrm{min} \text { (constant flow) (Schneider) }\end{array}$ & $1.2 \mathrm{~mL} / \mathrm{min}$ (constant flow) \\
\hline $\begin{array}{l}\text { Injector type } \\
\text { and temperature }\end{array}$ & $\begin{array}{l}\text { SSL, } \\
280^{\circ} \mathrm{C} \text { (da Cunha) } \\
250^{\circ} \mathrm{C} \text { (Schneider) }\end{array}$ & $\mathrm{SSL}, 280^{\circ} \mathrm{C}$ \\
\hline $\begin{array}{l}\text { Split injection } \\
\text { ratio }\end{array}$ & $\begin{array}{l}\text { splitless (da Cunha) } \\
\text { 1:20 (Schneider) }\end{array}$ & splitless \\
\hline Injection volume & - & - \\
\hline $\begin{array}{l}\text { Temperature } \\
\text { program }\end{array}$ & $\begin{array}{l}60^{\circ} \mathrm{C}(1 \mathrm{~min}) \text {, then } 3{ }^{\circ} \mathrm{C} / \mathrm{min} \text { to } 280^{\circ} \mathrm{C}(25 \mathrm{~min}) \text { (da } \\
\text { Cunha) } \\
\left.40^{\circ} \mathrm{C} \text { ( } 0 \mathrm{~min}\right) \text {, then } 3{ }^{\circ} \mathrm{C} / \mathrm{min} \text { to } 120^{\circ} \mathrm{C} \text {, then } 2^{\circ} \mathrm{C} / \mathrm{min} \text { to } \\
200^{\circ} \mathrm{C} \text { then } 10^{\circ} \mathrm{C} / \mathrm{min} \text { to } 280^{\circ} \mathrm{C} \text { ( } 5 \mathrm{~min} \text { ) (Schneider) }\end{array}$ & $\begin{array}{l}60{ }^{\circ} \mathrm{C}(0.2 \mathrm{~min}) \text {, then } 3{ }^{\circ} \mathrm{C} / \mathrm{min} \text { to } 310{ }^{\circ} \mathrm{C}(20 \mathrm{~min}) \text {. The secondary } \\
\text { column temperature was } 10^{\circ} \mathrm{C} \text { above the temperature of the } \\
\text { main oven }\end{array}$ \\
\hline Columns & $\begin{array}{l}1^{\text {st }} \text { D: OV-5 }(5 \% \text { phenyl, } 95 \% \text { PDMS }) \\
(60 \mathrm{~m} \times 0.25 \mathrm{~mm} \times 0.10 \mu \mathrm{m}) \\
2^{\text {nd }} \mathrm{D}: \text { DB-17 } \mathrm{ms} \\
(50 \% \text { phenyl, } 50 \% \text { PDMS }) \\
(2.15 \mathrm{~m} \times 0.18 \mathrm{~mm} \times 0.18 \mu \mathrm{m})\end{array}$ & $\begin{array}{l}1^{\text {st }} \mathrm{D}: \text { DB-5 }(5 \% \text { phenyl, } 95 \% \text { PDMS }) \\
(50 \mathrm{~m} \times 0.25 \mathrm{~mm} \times 0.25 \mu \mathrm{m}) \\
2^{\text {nd }} \mathrm{D}: \text { DB-17 } \mathrm{ms} \\
(50 \% \text { phenyl, } 50 \% \text { PDMS }) \\
(2.15 \mathrm{~m} \times 0.18 \mathrm{~mm} \times 0.18 \mu \mathrm{m})\end{array}$ \\
\hline Ovens & - & 2 \\
\hline Modulation time & $6 \mathrm{~s}$ & $8 \mathrm{~s}$ \\
\hline Modulation & liquid $\mathrm{N}_{2}$ quad-jet modulator & liquid $\mathrm{N}_{2}$ quad-jet modulator \\
\hline $\begin{array}{l}\text { Transfer line } \\
\text { temp. }\end{array}$ & $250^{\circ} \mathrm{C}$ & $320^{\circ} \mathrm{C}$ \\
\hline Detector & MS & MS \\
\hline Mass analyzer & quadrupole & TOF (Pegasus IV, Leco) \\
\hline $\begin{array}{l}\text { Ionization } \\
\text { (temp.) }\end{array}$ & $\mathrm{El},+70 \mathrm{eV}, 250^{\circ} \mathrm{C}$ & $\mathrm{El},+70 \mathrm{eV}, 250^{\circ} \mathrm{C}$ \\
\hline Scanning range & - & $45-500(\mathrm{~m} / \mathrm{z})$ \\
\hline Acquisition rate & $52.6 \mathrm{~Hz}$ & $100 \mathrm{~Hz}$ \\
\hline Quantification & RPV & RPV \\
\hline
\end{tabular}


Table S2e Other research groups

\begin{tabular}{|c|c|c|c|}
\hline & Omais et al. ${ }^{39}$ & Mullen et al. ${ }^{49}$ & Shashkov and Sidelnikov ${ }^{52}$ \\
\hline Published & 2013 & 2013 & 2016 \\
\hline Method & $\begin{array}{l}\mathrm{GC} \times \mathrm{GC}-\mathrm{TOF}-\mathrm{MS} \\
\mathrm{GC} \times \mathrm{GC}-\mathrm{FID}\end{array}$ & $\mathrm{GC} \times \mathrm{GC}-\mathrm{qMS}$ & $\mathrm{GC} \times \mathrm{GC}-\mathrm{FID}$ \\
\hline Column configuration & $\begin{array}{l}\mathrm{NP} \\
\mathrm{RP}\end{array}$ & $\mathrm{RP}$ & $\mathrm{RP}$ \\
\hline Sample treatment & ethyl acetate & methanol & not specified \\
\hline Internal standard & no & no & no \\
\hline Gas chromatograph & See the manuscript & QC-2010 (Shimadzu) & 7890 A (Agilent) \\
\hline Carrier gas & Helium & Helium & $\begin{array}{l}\left.\text { Helium ( } 1^{\text {st }} \text { column }\right) \\
\text { Hydrogen }\left(2^{\text {nd }} \text { column }\right)\end{array}$ \\
\hline Carrier gas flow rate & See the manuscript & $3 \mathrm{~mL} / \mathrm{min}$ & $\begin{array}{l}0.65 \mathrm{~mL} / \mathrm{min}\left(1^{\text {st }}\right) \\
23 \mathrm{~mL} / \mathrm{min}\left(2^{\text {nd }}\right)\end{array}$ \\
\hline Injector type and temperature & $\mathrm{SSL} / 320^{\circ} \mathrm{C}$ & SSL/- & $\mathrm{SSL} / 270^{\circ} \mathrm{C}$ \\
\hline Split injection ratio & $1: 100$ & $1: 30$ & - \\
\hline Injection volume & $0.3 \mu \mathrm{L}$ & - & - \\
\hline Temperature program & $\begin{array}{l}30^{\circ} \mathrm{C} \text {, then } 2^{\circ} \mathrm{C} / \mathrm{min} \text { to the } \\
\text { maximum allowed by the } \\
\text { columns }\end{array}$ & $\begin{array}{l}45^{\circ} \mathrm{C}(4 \mathrm{~min}) \text {, then } 3{ }^{\circ} \mathrm{C} / \mathrm{min} \\
\text { to } 280^{\circ} \mathrm{C}(20 \mathrm{~min})\end{array}$ & $\begin{array}{l}70{ }^{\circ} \mathrm{C}(3 \mathrm{~min}), \text { then } \\
8{ }^{\circ} \mathrm{C} / \mathrm{min} \text { to } 270{ }^{\circ} \mathrm{C}\end{array}$ \\
\hline Columns & $\begin{array}{l}\text { various column } \\
\text { combinations, } \\
\text { see the manuscript }\end{array}$ & $\begin{array}{l}1^{\text {st }} \text { D: DB-1701 (14 \% CPP, } \\
86 \% \text { PDMS }) \\
(60 \mathrm{~m} \times 0.25 \mathrm{~mm} \times 0.25 \mu \mathrm{m}) \\
2^{\text {nd }} \text { D: DB-5 ms } \\
(5 \% \text { phenyl, } 95 \% \text { PDMS }) \\
(2 \mathrm{~m} \times 0.25 \mathrm{~mm} \times 0.25 \mu \mathrm{m}) \\
\end{array}$ & see the manuscript \\
\hline Ovens & 1 & 1 & 1 \\
\hline Modulation time (s) & see the manuscript & 4 & 1.5 \\
\hline Modulation & - & Zoex ZX-2 LN 2 -cooled-loop & Zoex ZX-2 LN LN$_{2}$-cooled-loop \\
\hline Interface temp. & - & - & - \\
\hline Detector & MS & MS & $\operatorname{FID}\left(270^{\circ} \mathrm{C}\right)$ \\
\hline Mass analyzer & TOF (Pegasus IV, Leco) & quadrupole & - \\
\hline Ionization (temp.) & - & - & - \\
\hline Scanning range $(\mathrm{m} / \mathrm{z})$ & $45-750$ & $35-400.5$ & - \\
\hline Acquisition rate & $100 \mathrm{~Hz}$ & $20 \mathrm{~Hz}$ & - \\
\hline Quantification & $\begin{array}{l}\text { FID - measured and } \\
\text { quantified response factors }\end{array}$ & qMS - RPV & no \\
\hline
\end{tabular}


Table S2e Other research groups (continued)

\begin{tabular}{|c|c|c|c|c|}
\hline & Hung et al. ${ }^{51}$ & Olcese et al. ${ }^{53}$ & Ware et al. ${ }^{46}$ & Fullana et al. ${ }^{44}$ \\
\hline Published & 2020 & 2013 & 2017 & 2005 \\
\hline Method & GC $\times$ GC-Orbitrap-MS & GC-GC-FID & $\mathrm{GC} \times \mathrm{GC}-\mathrm{TOF}-\mathrm{MS}$ & $\mathrm{GC} \times \mathrm{GC}-\mathrm{TOF}-\mathrm{MS}$ \\
\hline $\begin{array}{l}\text { Column } \\
\text { configuration }\end{array}$ & $\mathrm{RP}$ & NP & NP & NP \\
\hline $\begin{array}{l}\text { Sample } \\
\text { treatment }\end{array}$ & $\operatorname{THF}(1: 1 \mathrm{w} / \mathrm{w})$ & methanol & $\mathrm{DCM}, 30 \mathrm{mg} / \mathrm{mL}$ & DCM \\
\hline $\begin{array}{l}\text { Internal } \\
\text { standard }\end{array}$ & $\alpha$-pinene & 1-undecene & - & see the manuscript \\
\hline $\begin{array}{l}\text { Gas } \\
\text { chromatograph }\end{array}$ & $\begin{array}{l}\text { Trace } 1310 \text { GC (Thermo- } \\
\text { Fisher Scientific) }\end{array}$ & 7890 (Agilent) & NS & HP 5890N (Agilent) \\
\hline Carrier gas & Helium & Helium & Helium & Helium \\
\hline $\begin{array}{l}\text { Carrier gas flow } \\
\text { rate }\end{array}$ & $1.5 \mathrm{~mL} / \mathrm{min}$ & - & $1 \mathrm{~mL} / \mathrm{min}$ & - \\
\hline $\begin{array}{l}\text { Injector type } \\
\text { and } \\
\text { temperature }\end{array}$ & $\mathrm{SS} / 250^{\circ} \mathrm{C}$ & - & $\mathrm{SS} / 300^{\circ} \mathrm{C}$ & - \\
\hline $\begin{array}{l}\text { Split injection } \\
\text { ratio }\end{array}$ & NS & - & $1: 5$ & - \\
\hline $\begin{array}{l}\text { Injection } \\
\text { volume }\end{array}$ & NS & - & $1 \mu \mathrm{L}$ & - \\
\hline $\begin{array}{l}\text { Temperature } \\
\text { program }\end{array}$ & $\begin{array}{l}55^{\circ} \mathrm{C}(2 \mathrm{~min}), \text { then } 5^{\circ} \mathrm{C} / \mathrm{min} \\
\text { to } 240^{\circ} \mathrm{C}(5 \mathrm{~min})\end{array}$ & $\begin{array}{l}40{ }^{\circ} \mathrm{C}(10 \mathrm{~min}), \text { then } \\
5{ }^{\circ} \mathrm{C} / \mathrm{min} \text { to } 200^{\circ} \mathrm{C}(21 \mathrm{~min})\end{array}$ & $\begin{array}{l}60^{\circ} \mathrm{C}(0.5 \mathrm{~min}), \text { then } \\
3{ }^{\circ} \mathrm{C} / \mathrm{min} \text { to } 340^{\circ} \mathrm{C}\end{array}$ & $\begin{array}{l}40{ }^{\circ} \mathrm{C}(15 \mathrm{~min}) \text {, then } 4{ }^{\circ} \mathrm{C} / \mathrm{min} \\
\text { to } 260^{\circ} \mathrm{C}(15 \mathrm{~min})\end{array}$ \\
\hline Columns & $\begin{array}{l}1^{\text {st }} \text { D: FFAP }(100 \% \text { acid } \\
\text { modified PEG) } \\
(50 \mathrm{~m} \times 0.25 \mathrm{~mm} \times 0.25 \mu \mathrm{m}) \\
2^{\text {nd }} \text { D: SLB-5 }(5 \% \text { phenyl, } \\
95 \% \text { PDMS }) \\
(2 \mathrm{~m} \times 0.10 \mathrm{~mm} \times 0.10 \mu \mathrm{m})\end{array}$ & $\begin{array}{l}1^{\text {st }} \text { D: HP-5MS (5 \% phenyl, } \\
95 \% \text { PDMS) } \\
(30 \mathrm{~m} \times 0.25 \mathrm{~mm} \times 0.25 \mu \mathrm{m}) \\
2^{\text {nd }} \mathrm{D}: \text { DB-Wax }(100 \% \text { PEG) } \\
(30 \mathrm{~m} \times 0.32 \mathrm{~mm} \times 0.25 \mu \mathrm{m})\end{array}$ & $\begin{array}{l}1^{\text {st }} \text { D: BP-1 }(100 \% \text { PDMS }) \\
(60 \mathrm{~m} \times 0.25 \mathrm{~mm} \times 0.25 \mu \mathrm{m}) \\
2^{\text {nd }} \mathrm{D}: \text { BPX-50 } \\
(50 \% \text { phenyl, } 50 \% \text { PSPS }) \\
(1.4 \mathrm{~m} \times 0.25 \mathrm{~mm} \times 0.10 \mu \mathrm{m})\end{array}$ & $\begin{array}{l}1^{\text {st }} \text { D: MXT-5 (5\% phenyl, } \\
95 \% \text { PDMS) } \\
(30 \mathrm{~m} \times 0.53 \mathrm{~mm} \times 1 \mu \mathrm{m}) \\
2^{\text {nd }} \text { D: BP20 } \\
(100 \% \text { PEG }) \\
(2.5 \mathrm{~m} \times 0.10 \mathrm{~mm} \times 0.10 \mu \mathrm{m})\end{array}$ \\
\hline Ovens & 2 & 1 & 2 & - \\
\hline $\begin{array}{l}\text { Modulation } \\
\text { time (s) }\end{array}$ & 5 & - & 6 & 20 \\
\hline Modulation & $\begin{array}{l}\text { two-stage loop-type } \\
\text { modulator }\end{array}$ & Dean's switch walve & - & - \\
\hline Interface temp. & - & - & - & - \\
\hline Detector & $\begin{array}{l}\text { Thermo Scientific Q } \\
\text { Exactive GC hybrid } \\
\text { quadrupole-Orbitrap }\end{array}$ & MS, FID & MS & MS \\
\hline Mass analyzer & Orbitrap-MS & - & TOF (Pegasus IV, Leco) & TOF (Pegasus III, Leco) \\
\hline $\begin{array}{l}\text { Ionization } \\
\text { (temp.) }\end{array}$ & $230^{\circ} \mathrm{C}$ & - & - & - \\
\hline $\begin{array}{l}\text { Scanning range } \\
(\mathrm{m} / \mathrm{z})\end{array}$ & $50-450$ & - & - & $40-300$ \\
\hline $\begin{array}{l}\text { Acquisition } \\
\text { rate }(\mathrm{Hz})\end{array}$ & $25 \mathrm{~Hz} / 43 \mathrm{~Hz}$ & - & - & 100 \\
\hline Quantification & $\begin{array}{l}23 \text { compounds, calibration } \\
\text { curves }\end{array}$ & FID - response factors & FID & FID \\
\hline
\end{tabular}


Table S2e Other research groups (continued)

\begin{tabular}{|c|c|c|c|c|c|c|}
\hline & Karp et al. ${ }^{42}$ & Wang et al. ${ }^{48}$ & $\begin{array}{l}\text { Eschenbacher et al. } \\
\qquad 40,41\end{array}$ & $\underset{50}{\text { Margeriat et al. }}$ & Mao et al. ${ }^{47}$ & $\begin{array}{l}\text { Johansson et al. } \\
43\end{array}$ \\
\hline Published & 2016 & 2020 & 2020 & 2018 & 2018 & 2017 \\
\hline Method & $\mathrm{GC} \times \mathrm{GC}-\mathrm{TOF}-\mathrm{MS}$ & $\begin{array}{l}\text { GC } \times \text { GC-TOF }- \\
\text { MS }\end{array}$ & $\begin{array}{l}\text { GC } \times \text { GC-TOF- } \\
\text { MS/FID }\end{array}$ & $\begin{array}{l}\mathrm{GC} \times \mathrm{GC}- \\
\mathrm{qMS} / \mathrm{FID}\end{array}$ & $\begin{array}{l}\text { GC } \times \text { GC-TOF }- \\
\text { MS }\end{array}$ & $\begin{array}{l}\text { GC } \times \text { GC-TOF }- \\
\text { MS }\end{array}$ \\
\hline $\begin{array}{l}\text { Column } \\
\text { configuration }\end{array}$ & $\mathrm{RP}$ & NP & $\mathrm{RP}$ & $\mathrm{RP}$ & NP & NP \\
\hline $\begin{array}{l}\text { Sample } \\
\text { treatment }\end{array}$ & pyridine & chloroform & 1:1 vol. in THF & DCM & DCM & $20 \mathrm{mg} / \mathrm{mL} \mathrm{ACN}$ \\
\hline $\begin{array}{l}\text { Internal } \\
\text { standard }\end{array}$ & $\begin{array}{l}\text { cyclohexane } \\
\text { carboxylic acid }\end{array}$ & Dibutyl ether & no & n-decane & no & $\begin{array}{l}\text { 2-fluoro-1,1- } \\
\text { biphenyl }\end{array}$ \\
\hline $\begin{array}{l}\text { Gas } \\
\text { chromatograph }\end{array}$ & LECO Pegasus & 7890A (Agilent) & 7890A (Agilent) & 6890N (Agilent) & - & LECO Pegasus \\
\hline Carrier gas & Helium & Helium (5.0) & Helium & Helium & - & Helium \\
\hline $\begin{array}{l}\text { Carrier gas flow } \\
\text { rate }\end{array}$ & $1 \mathrm{~mL} / \mathrm{min}$ & $1 \mathrm{~mL} / \mathrm{min}$ & $1.5 \mathrm{~mL} / \mathrm{min}$ & - & - & - \\
\hline $\begin{array}{l}\text { Injector type } \\
\text { and temperature }\end{array}$ & $\mathrm{SSL}, 300^{\circ} \mathrm{C}$ & $\mathrm{SSL}, 280^{\circ} \mathrm{C}$ & $\begin{array}{l}\text { PTV, } 40 \text { to } 300, \\
10^{\circ} \mathrm{C} / \mathrm{s}\end{array}$ & - & - & $\mathrm{SSL}, 300^{\circ} \mathrm{C}$ \\
\hline $\begin{array}{l}\text { Split injection } \\
\text { ratio }\end{array}$ & $1: 30$ & $1: 5$ & $1: 100$ & - & - & $1: 50$ \\
\hline Injection volume & $2 \mu \mathrm{L}$ & $1 \mu \mathrm{L}$ & $0.3 \mu \mathrm{L}$ & - & - & - \\
\hline $\begin{array}{l}\text { Temperature } \\
\text { program }\end{array}$ & $\begin{array}{l}40{ }^{\circ} \mathrm{C}(1 \mathrm{~min}) \text {, then } \\
7.5^{\circ} \mathrm{C} / \mathrm{min} \text { to } 155^{\circ} \mathrm{C} \text {, } \\
\text { then } 15^{\circ} \mathrm{C} / \mathrm{min} \text { to } \\
225^{\circ} \mathrm{C}(20 \mathrm{~min}) \\
\text { Second oven }+10{ }^{\circ} \mathrm{C}\end{array}$ & $\begin{array}{l}0{ }^{\circ} \mathrm{C}(4 \mathrm{~min}) \text {, } \\
\text { then } 3^{\circ} \mathrm{C} / \mathrm{min} \\
\text { to } 240^{\circ} \mathrm{C} \text {, then } \\
20{ }^{\circ} \mathrm{C} / \mathrm{min} \text { to } \\
300^{\circ} \mathrm{C} \\
\text { Second oven } \\
+5{ }^{\circ} \mathrm{C}\end{array}$ & $\begin{array}{l}35^{\circ} \mathrm{C}(1.5 \mathrm{~min}) \text {, } \\
\text { then } 3^{\circ} \mathrm{C} / \mathrm{min} \text { to } \\
275^{\circ} \mathrm{C} \\
\text { Second oven }+10^{\circ} \mathrm{C}\end{array}$ & $\begin{array}{l}\text { First oven } \\
50{ }^{\circ} \mathrm{C}(5 \mathrm{~min}) \text {, } \\
\text { then } \\
1.75{ }^{\circ} \mathrm{C} / \mathrm{min} \text { to } \\
300^{\circ} \mathrm{C} \\
\text { Second oven } \\
50{ }^{\circ} \mathrm{C}(0 \mathrm{~min}) \text {, } \\
\text { then } 1.8^{\circ} \mathrm{C} / \mathrm{min} \\
\text { to } 320^{\circ} \mathrm{C}\end{array}$ & - & $\begin{array}{l}40{ }^{\circ} \mathrm{C}(4 \mathrm{~min}) \text {, } \\
\text { then } 15^{\circ} \mathrm{C} / \mathrm{min} \\
\text { to } 245^{\circ} \mathrm{C} \\
(15 \mathrm{~min}) \\
\text { Second oven } \\
+10^{\circ} \mathrm{C}\end{array}$ \\
\hline Columns & $\begin{array}{l}\text { RTX-5 }(10 \mathrm{~m}) / \mathrm{DB}- \\
1701(0.75 \mathrm{~m})\end{array}$ & $\begin{array}{l}\text { DB- } \\
5 \mathrm{~ms}(30 \mathrm{~m}) / \mathrm{DB}- \\
17 \mathrm{HT}(2 \mathrm{~m})\end{array}$ & Rtx-1701/BPX5 & $\begin{array}{l}\text { VF1701 } \\
(30 \mathrm{~m}) / \mathrm{DB} 1(2 \mathrm{~m})\end{array}$ & $\begin{array}{l}\text { Solgelwax } \\
(30 \mathrm{~m}) / \text { Rxi-5Sil } \\
\text { MS }(2 \mathrm{~m})\end{array}$ & $\begin{array}{l}\text { RTX-5(10m)/DB- } \\
1701(0.75 \mathrm{~m})\end{array}$ \\
\hline Ovens & 2 & 2 & 2 & 2 & 1 & 2 \\
\hline Modulation time & $4 \mathrm{~s}$ & $8 \mathrm{~s}$ & $7 \mathrm{~s}$ & $12 \mathrm{~s}$ & - & $4 \mathrm{~s}$ \\
\hline Modulation & - & - & $\begin{array}{l}\text { a quad-jet, dual- } \\
\text { stage cryogenic- } \\
\text { based (liquid N2) }\end{array}$ & $\begin{array}{l}\text { cryogenic } \\
\text { modulator } \\
\text { (liquid N2) }\end{array}$ & - & - \\
\hline Interface temp. & $250^{\circ} \mathrm{C}$ & $270^{\circ} \mathrm{C}$ & $250^{\circ} \mathrm{C}$ & - & - & - \\
\hline Detector & - & $\begin{array}{l}\text { Pegasus IV } \\
\text { (Leco) }\end{array}$ & Pegasus IV (Leco) & 5975B (Agilent) & $\begin{array}{l}\text { Pegasus IV } \\
\text { (Leco) }\end{array}$ & $\begin{array}{l}\text { Pegasus IV } \\
\text { (Leco) }\end{array}$ \\
\hline Mass analyzer & TOF-MS & TOF & TOF & quadrupole & TOF & TOF \\
\hline $\begin{array}{l}\text { Ionization } \\
\text { (temp.) }\end{array}$ & - & $220^{\circ} \mathrm{C}$ & $225^{\circ} \mathrm{C}$ & - & - & - \\
\hline $\begin{array}{l}\text { Scanning range } \\
(\mathrm{m} / \mathrm{z})\end{array}$ & $29-350$ & $33-550$ & $41-441$ & $45-300$ & - & $29-350$ \\
\hline Acquisition rate & $200 \mathrm{~Hz}$ & $100 \mathrm{~Hz}$ & $100 \mathrm{~Hz}$ & $22 \mathrm{~Hz}$ & - & $200 \mathrm{~Hz}$ \\
\hline Quantification & 61 standards & $\begin{array}{l}\text { TOF-MS - RPV } \\
\text { and IS method }\end{array}$ & FID - RPV & FID - ECN & TOF-MS - RPV & - \\
\hline
\end{tabular}




\section{References}

1. Sfetsas, T.; Michailof, C.; Lappas, A.; Li, Q.; Kneale, B., Qualitative and quantitative analysis of pyrolysis oil by gas chromatography with flame ionization detection and comprehensive two-dimensional gas chromatography with time-of-flight mass spectrometry. Journal of Chromatography A 2011, 1218 (21), 3317-3325.

2. Michailof, C.; Sfetsas, T.; Stefanidis, S.; Kalogiannis, K.; Theodoridis, G.; Lappas, A., Quantitative and qualitative analysis of hemicellulose, cellulose and lignin bio-oils by comprehensive two-dimensional gas chromatography with time-of-flight mass spectrometry. Journal of Chromatography A 2014, 1369, 147-160.

3. Iliopoulou, E.; Mihailof, C.; Kalogiannis, K.; Pachnos, M.; Emmanouilidou, M.; Lappas, A. In Characterization studies of Waste-bio-derived feedstocks, Symbiosis International Conference, Athens, Greece, 2014.

4. Kalogiannis, K. G.; Stefanidis, S. D.; Michailof, C. M.; Lappas, A. A., Castor bean cake residues upgrading towards high added value products via fast catalytic pyrolysis. Biomass and Bioenergy 2016, 95, 405-415.

5. Kalogiannis, K. G.; Stefanidis, S. D.; Michailof, C. M.; Lappas, A. A.; Sjöholm, E., Pyrolysis of lignin with 2DGC quantification of lignin oil: Effect of lignin type, process temperature and ZSM-5 in situ upgrading. Journal of Analytical and Applied Pyrolysis 2015, 115, 410-418.

6. Stefanidis, S. D.; Heracleous, E.; Patiaka, D. T.; Kalogiannis, K. G.; Michailof, C. M.; Lappas, A. A., Optimization of bio-oil yields by demineralization of low quality biomass. Biomass and Bioenergy 2015, 83, 105-115.

7. Osatiashtiani, A.; Puértolas, B.; Oliveira, C. C. S.; Manayil, J. C.; Barbero, B.; Isaacs, M.; Michailof, C.; Heracleous, E.; Pérez-Ramírez, J.; Lee, A. F.; Wilson, K., On the influence of Si:Al ratio and hierarchical porosity of FAU zeolites in solid acid catalysed esterification pretreatment of bio-oil. Biomass Conversion and Biorefinery 2017, 7 (3), 331-342.

8. Marsman, J.; Wildschut, J.; Mahfud, F.; Heeres, H., Identification of components in fast pyrolysis oil and upgraded products by comprehensive two-dimensional gas chromatography and flame ionisation detection. Journal of Chromatography A 2007, 1150 (1-2), 21-27.

9. Marsman, J.; Wildschut, J.; Evers, P.; de Koning, S.; Heeres, H., Identification and classification of components in flash pyrolysis oil and hydrodeoxygenated oils by two-dimensional gas chromatography and time-of-flight mass spectrometry. Journal of Chromatography A 2008, 1188 (1), 17-25.

10. Kloekhorst, A.; Wildschut, J.; Heeres, H. J., Catalytic hydrotreatment of pyrolytic lignins to give alkylphenolics and aromatics using a supported Ru catalyst. Catal. Sci. Technol. 2014, 4 (8), 2367-2377.

11. Priharto, N.; Ronsse, F.; Prins, W.; Hita, I.; Deuss, P. J.; Heeres, H. J., Hydrotreatment of pyrolysis liquids derived from second-generation bioethanol production residues over NiMo and CoMo catalysts. Biomass and Bioenergy 2019, 126, 84-93.

12. Priharto, N.; Ronsse, F.; Yildiz, G.; Heeres, H. J.; Deuss, P. J.; Prins, W., Fast pyrolysis with fractional condensation of lignin-rich digested stillage from second-generation bioethanol production. Journal of Analytical and Applied Pyrolysis 2020, 145.

13. Priharto, N.; Ronsse, F.; Prins, W.; Carleer, R.; Heeres, H. J., Experimental studies on a two-step fast pyrolysiscatalytic hydrotreatment process for hydrocarbons from microalgae (Nannochloropsis gaditana and Scenedesmus almeriensis). Fuel Processing Technology 2020, 206, 106466.

14. Figueirêdo, M. B.; Jotic, Z.; Deuss, P. J.; Venderbosch, R. H.; Heeres, H. J., Hydrotreatment of pyrolytic lignins to aromatics and phenolics using heterogeneous catalysts. Fuel Processing Technology 2019, 189, 28-38.

15. Figueirêdo, M. B.; Deuss, P. J.; Venderbosch, R. H.; Heeres, H. J., Catalytic hydrotreatment of pyrolytic lignins from different sources to biobased chemicals: Identification of feed-product relations. Biomass and Bioenergy 2020, 134.

16. Figueirêdo, M. B.; Venderbosch, R. H.; Heeres, H. J.; Deuss, P. J., In-depth structural characterization of the lignin fraction of a pine-derived pyrolysis oil. Journal of Analytical and Applied Pyrolysis 2020, 149.

17. Ghysels, S.; Dubuisson, B.; Mehmet, P.; Rohrbach, L.; Van den Bulcke, J.; Heeres, H. J.; Ronsse, F., Improving fast pyrolysis of lignin using three additives with different modes of action. Green Chemistry 2020, 22, 6471.

18. Djokic, M. R.; Dijkmans, T.; Yildiz, G.; Prins, W.; Van Geem, K. M., Quantitative analysis of crude and stabilized bio-oils by comprehensive two-dimensional gas-chromatography. Journal of Chromatography A 2012, 1257 (0), 131-140. 
19. Negahdar, L.; Gonzalez-Quiroga, A.; Otyuskaya, D.; Toraman, H. E.; Liu, L.; Jastrzebski, J. T.; Van Geem, K. M.; Marin, G. B.; Thybaut, J. W.; Weckhuysen, B. M., Characterization and comparison of fast pyrolysis bio-oils from pinewood, rapeseed cake, and wheat straw using $13 \mathrm{C}$ NMR and comprehensive GC $\times$ GC. ACS Sustainable Chemistry \& Engineering 2016, 4 (9), 4974-4985.

20. Toraman, H. E.; Franz, K.; Ronsse, F.; Van Geem, K. M.; Marin, G. B., Quantitative analysis of nitrogen containing compounds in microalgae based bio-oils using comprehensive two-dimensional gas-chromatography coupled to nitrogen chemiluminescence detector and time of flight mass spectrometer. Journal of Chromatography A 2016, 1460, 135-146.

21. Tessarolo, N. S.; dos Santos, L. R.; Silva, R. S.; Azevedo, D. A., Chemical characterization of bio-oils using comprehensive two-dimensional gas chromatography with time-of-flight mass spectrometry. Journal of Chromatography A 2013, 1279, 68-75.

22. Tessarolo, N. S.; Silva, R. C.; Vanini, G.; Pinho, A.; Romão, W.; de Castro, E. V.; Azevedo, D. A., Assessing the chemical composition of bio-oils using FT-ICR mass spectrometry and comprehensive two-dimensional gas chromatography with time-of-flight mass spectrometry. Microchemical Journal 2014, 117, 68-76.

23. Tessarolo, N. S.; Silva, R. V.; Vanini, G.; Casilli, A.; Ximenes, V. L.; Mendes, F. L.; de Rezende Pinho, A.; Romão, W.; de Castro, E. V.; Kaiser, C. R. J. J. o. a.; pyrolysis, a., Characterization of thermal and catalytic pyrolysis biooils by high-resolution techniques: 1H NMR, GCX GC-TOFMS and FT-ICR MS. 2016, 117, 257-267.

24. Silva, R. V.; Casilli, A.; Sampaio, A. L.; Ávila, B. M.; Veloso, M. C.; Azevedo, D. A.; Romeiro, G. A., The analytical characterization of castor seed cake pyrolysis bio-oils by using comprehensive GC coupled to time of flight mass spectrometry. Journal of Analytical Applied Pyrolysis 2014, 106, 152-159.

25. Silva, R. V. S.; Tessarolo, N. S.; Pereira, V. B.; Ximenes, V. L.; Mendes, F. L.; de Almeida, M. B. B.; Azevedo, D. A., Quantification of real thermal, catalytic, and hydrodeoxygenated bio-oils via comprehensive two-dimensional gas chromatography with mass spectrometry. Talanta 2017, 164, 626-635.

26. Mendes, F. L.; Ximenes, V. L.; de Almeida, M. B. B.; Azevedo, D. A.; Tessarolo, N. S.; de Rezende Pinho, A., Catalytic pyrolysis of sugarcane bagasse and pinewood in a pilot scale unit. Journal of Analytical and Applied Pyrolysis 2016, 122, 395-404.

27. Moraes, M. S. A.; Migliorini, M. V.; Damasceno, F. C.; Georges, F.; Almeida, S.; Zini, C. A.; Jacques, R. A.; Caramão, E. B., Qualitative analysis of bio oils of agricultural residues obtained through pyrolysis using comprehensive two dimensional gas chromatography with time-of-flight mass spectrometric detector. Journal of Analytical and Applied Pyrolysis 2012, 98, 51-64.

28. Moraes, M. S. A.; Georges, F.; Almeida, S. R.; Damasceno, F. C.; Maciel, G. P. d. S.; Zini, C. A.; Jacques, R. A.; Caramão, E. B., Analysis of products from pyrolysis of Brazilian sugar cane straw. Fuel Processing Technology 2012, 101, 35-43.

29. Faccini, C. S.; Vecchia, I. D.; Ribeiro, D.; Zini, C. A.; Caramão, E. B., Comprehensive Two-Dimensional GC with TOF-MS Detection: Study of Pyrolytic Bio-Oil of Kraft Mill Residues. Journal of the Brazilian Chemical Society 2013.

30. Onorevoli, B.; Machado, M. E.; Polidoro, A. d. S.; Corbelini, V. A.; Caramão, E. B.; Jacques, R. A., Pyrolysis of Residual Tobacco Seeds: Characterization of Nitrogen Compounds in Bio-oil Using Comprehensive TwoDimensional Gas Chromatography with Mass Spectrometry Detection. Energy \& Fuels 2017, 31 (9), 9402-9407.

31. Primaz, C. T.; Schena, T.; Lazzari, E.; Caramão, E. B.; Jacques, R. A., Influence of the temperature in the yield and composition of the bio-oil from the pyrolysis of spent coffee grounds: Characterization by comprehensive two dimensional gas chromatography. Fuel 2018, 232, 572-580.

32. Schena, T.; Bjerk, T. R.; von Muhlen, C.; Caramao, E. B., Influence of acquisition rate on performance of fast comprehensive two-dimensional gas chromatography coupled with time-of-flight mass spectrometry for coconut fiber bio-oil characterization. Talanta 2020, 219, 121186.

33. Schena, T.; Farrapeira, R.; Bjerk, T. R.; Krause, L. C.; Mühlen, C.; Caramão, E. B., Fast two-dimensional gas chromatography applied in the characterization of bio-oil from the pyrolysis of coconut fibers. Separation Science Plus 2019, 2 (3), 89-99.

34. Lazzari, E.; Souza Silva, É. A.; Bjerk, T. R.; Schneider, J. K.; Bastos Caramão, E., Evaluation of the matrix effect in the quantitative bio-oil analysis by gas chromatography. Fuel 2021, 290, 119866. 
35. Torri, I. D.; Paasikallio, V.; Faccini, C. S.; Huff, R.; Caramao, E. B.; Sacon, V.; Oasmaa, A.; Zini, C. A., Bio-oil production of softwood and hardwood forest industry residues through fast and intermediate pyrolysis and its chromatographic characterization. Bioresour Technol 2016, 200, 680-90.

36. da Cunha, M. E.; Schneider, J. K.; Brasil, M. C.; Cardoso, C. A.; Monteiro, L. R.; Mendes, F. L.; Pinho, A.; Jacques, R. A.; Machado, M. E.; Freitas, L. S.; Caramão, E. B., Analysis of fractions and bio-oil of sugar cane straw by one-dimensional and two-dimensional gas chromatography with quadrupole mass spectrometry (GC×GC/qMS). Microchemical Journal 2013, 110, 113-119.

37. Pereira da Silva Maciel, G.; Machado, M. E.; Barbará, J. A.; Molin, D. D.; Caramão, E. B.; Jacques, R. A., $\mathrm{GC} \times \mathrm{GC} / \mathrm{TOFMS}$ analysis concerning the identification of organic compounds extracted from the aqueous phase of sugarcane straw fast pyrolysis oil. Biomass and Bioenergy 2016, 85, 198-206.

38. Schneider, J. K.; da Cunha, M. E.; dos Santos, A. L.; Maciel, G. P.; Brasil, M. C.; Pinho, A. R.; Mendes, F. L.; Jacques, R. A.; Caramão, E. B., Comprehensive two dimensional gas chromatography with fast-quadrupole mass spectrometry detector analysis of polar compounds extracted from the bio-oil from the pyrolysis of sawdust. Journal of Chromatography A 2014, 1356, 236-240.

39. Omais, B.; Crepier, J.; Charon, N.; Courtiade, M.; Quignard, A.; Thiebaut, D., Oxygen speciation in upgraded fast pyrolysis bio-oils by comprehensive two-dimensional gas chromatography. Analyst 2013, 138 (8), 2258-68.

40. Eschenbacher, A.; Saraeian, A.; Jensen, P. A.; Shanks, B. H.; Li, C.; Duus, J. Ø.; Smitshuysen, T. E. L.; Damsgaard, C. D.; Hansen, A. B.; Kling, K. I.; Mentzel, U. V.; Henriksen, U. B.; Ahrenfeldt, J.; Jensen, A. D., Deoxygenation of wheat straw fast pyrolysis vapors over Na-Al2O3 catalyst for production of bio-oil with low acidity. Chemical Engineering Journal 2020, 394.

41. Eschenbacher, A.; Saraeian, A.; Shanks, B. H.; Jensen, P. A.; Li, C.; Duus, J. Ø.; Hansen, A. B.; Mentzel, U. V.; Henriksen, U. B.; Ahrenfeldt, J.; Jensen, A. D., Enhancing bio-oil quality and energy recovery by atmospheric hydrodeoxygenation of wheat straw pyrolysis vapors using Pt and Mo-based catalysts. Sustainable Energy \& Fuels 2020, 4 (4), 1991-2008.

42. Karp, E. M.; Nimlos, C. T.; Deutch, S.; Salvachúa, D.; Cywar, R. M.; Beckham, G. T., Quantification of acidic compounds in complex biomass-derived streams. Green Chemistry 2016, 18 (17), 4750-4760.

43. Johansson, A.-C.; lisa, K.; Sandström, L.; Ben, H.; Pilath, H.; Deutch, S.; Wiinikka, H.; Öhrman, O. G. W., Fractional condensation of pyrolysis vapors produced from Nordic feedstocks in cyclone pyrolysis. Journal of Analytical and Applied Pyrolysis 2017, 123, 244-254.

44. Fullana, A.; Contreras, J. A.; Striebich, R. C.; Sidhu, S. S., Multidimensional GC/MS analysis of pyrolytic oils. Journal of Analytical and Applied Pyrolysis 2005, 74 (1), 315-326.

45. Rathsack, P.; Reichel, D.; Krzack, S.; Otto, M., Komprehensive Gaschromatographie-Massenspektrometrie von Alkylbenzolen in Pyrolyseölen aus Biomasse und Kohle. Chemie Ingenieur Technik 2014, 86 (10), 1779-1789.

46. Ware, R. L.; Rowland, S. M.; Rodgers, R. P.; Marshall, A. G., Advanced Chemical Characterization of Pyrolysis Oils from Landfill Waste, Recycled Plastics, and Forestry Residue. Energy \& Fuels 2017, 31 (8), 8210-8216.

47. Mao, L.; Li, Y.; Zhang, Z. C., Upgrading of derived pyrolysis vapors for the production of biofuels from corncobs. Frontiers of Chemical Science and Engineering 2018, 12 (1), 50-58.

48. Wang, Y.; Yin, R.; Chai, M.; Li, C.; Sarker, M.; Liu, R., Comparative study by GCX GC-TOFMS on the composition of crude and composite-additives bio-oil before and after accelerated aging treatment. Journal of the Energy Institute 2020.

49. Mullen, C. A.; Boateng, A. A.; Reichenbach, S. E., Hydrotreating of fast pyrolysis oils from protein-rich pennycress seed presscake. Fuel 2013, 111, 797-804.

50. Margeriat, A.; Bouzeggane, A.; Lorentz, C.; Laurenti, D.; Guilhaume, N.; Mirodatos, C.; Geantet, C.; Schuurman, Y., Catalytic conversion of beech wood pyrolytic vapors. Journal of Analytical and Applied Pyrolysis 2018, 130, 149-158.

51. Hung, N. V.; Mohabeer, C.; Vaccaro, M.; Marcotte, S.; Agasse-Peulon, V.; Abdelouahed, L.; Taouk, B.; Cardinael, P., Development of two-dimensional gas chromatography (GCxGC) coupled with Orbitrap-technologybased mass spectrometry: Interest in the identification of biofuel composition. J Mass Spectrom 2020, 55 (3), e4495. 
52. Shashkov, M. V.; Sidelnikov, V. N., Separation of phenol-containing pyrolysis products using comprehensive twodimensional chromatography with columns based on pyridinium ionic liquids. Journal of separation science 2016, 39 (19), 3754-3760.

53. Olcese, R.; Carré, V.; Aubriet, F. d. r.; Dufour, A., Selectivity of Bio-oils Catalytic Hydrotreatment Assessed by Petroleomic and GC* GC/MS-FID Analysis. Energy \& Fuels 2013, 27 (4), 2135-2145. 